\title{
उMR
}

\section{Regulatory role of microRNA184 in osteosarcoma cells}

\author{
G.R. Yin ${ }^{1,2}$, Q. Wang ${ }^{3}$, X.B. Zhang ${ }^{2}$ and S.J. Wang ${ }^{1}$ \\ 1'Department of Joint Surgery, The Second Hospital of Shandong University, \\ Jinan, China \\ 2Department of Orthopedics, DeZhou People's Hospital, DeZhou, China \\ ${ }^{3}$ DeZhou University, DeZhou, China \\ Corresponding author: S.J. Wang \\ E-mail: shaojin800987@163.com
}

Genet. Mol. Res. 14 (4): 14246-14252 (2015)

Received May 4, 2015

Accepted July 31, 2015

Published November 13, 2015

DOI http://dx.doi.org/10.4238/2015.November.13.8

\begin{abstract}
Osteosarcoma is a highly malignant cancer that often appears in teenagers. It is the most frequently occurring primary bone tumor, and can easily metastasize, resulting in high mortality. MicroRNAs express abnormally in osteosarcoma, and may function as oncogenes or tumor suppressors. Recent studies showed that microRNA184 (miR-184) is abnormally expressed in multiple tumors, and is involved in tumor cell growth, differentiation, invasion, and metastasis. Nevertheless, the role of miR-184 in osteosarcoma cells remains unknown. We evaluated the expression and function of microRNA184 in osteosarcoma cells. SOSP-M osteosarcoma cells were divided into normal control, miR-184 mimic, and miR-184 inhibitor groups. Real-time PCR was applied to detect miR184 expression. The 3-(4,5-dimethylthaizol-2-yl)-2,5-diphenyltetrazolium bromide assay was used to evaluate cell proliferation. Transwell assays were performed to detect changes in cell invasion ability. Compared with the control group, miR-184 expression was significantly increased in the miR-184 mimic group $(P<0.05)$. After miR-184 inhibitor transfection, miR-
\end{abstract}


184 expression was obviously reduced $(\mathrm{P}<0.05)$. Tumor cell proliferation was enhanced in the miR-184 mimic group $(P<0.05)$, whereas miR-184 inhibition suppressed cell proliferation $(P<0.05)$. Furthermore, tumor cell invasion increased after miR-184 mimic transfection $(P<0.05)$, and decreased after inhibiting miR-184 ( $\mathrm{P}<0.05)$. MiR-184 promotes tumor cell proliferation and invasion, and may represent a new biological target for osteosarcoma.

Key words: miR-184; Osteosarcoma; Proliferation; Invasion

\section{INTRODUCTION}

Osteosarcoma mainly occurs during the bone growth stage. Thus, it is common in children and adolescents. It is highly malignant, and is the most common cause of primary bone tumors. Pulmonary metastasis occurs in early stages of osteosarcoma, causing high mortality. Osteosarcoma originates from the mesenchymal tissue, and is characterized by spindle stromal cells and osteoid tissue (Vijayakumar et al., 2014; Trosman and Krakovitz, 2015). No obvious clinical symptoms occur in the early phase of osteosarcoma. Rather, it is usually misdiagnosed as growing pains or trauma, since it occurs during bone growth. Osteosarcoma progresses and metastasizes quickly, and is resistant to chemotherapy. Due to its high mortality rate, it can cause great pain and heavy economic burden to the patient and family (Ebrahimzadeh et al., 2013; Sun et al., 2015).

Current research suggests that osteosarcoma is associated with abnormal gene expression. The pathogenesis of osteosarcoma, including development and metastasis, involves multiple genes (Garajova et al., 2014; Phuah and Nagoor, 2014). MicroRNAs (miRNAs), which widely exist in animals and plants, are non-coding, small, regulatory RNAs (Orang and Barzegari, 2014; Saadatian et al., 2014) that can negatively regulate gene expression. They function via complete or incomplete pairing with target genes, or by inhibiting the downstream transcription of target proteins, mRNA degradation, and protein translation (Gandhi et al., 2014). MiRNAs have numerous functions, affecting growth, metabolism, the endocrine system, hormone secretion, and embryonic stem cells, thereby regulating adaption to the environment. MiRNAs are closely associated with tumor occurrence and development. Some miRNAs can promote tumor proliferation and metastasis, whereas others inhibit tumorigenesis (Gallach et al., 2014; Lindner et al., 2015). Previous research showed that miRNAs are abnormally expressed in osteosarcoma, and may function as oncogenes or tumor suppressors. Recent studies suggest that microRNA184 (miR-184) is abnormally expressed in various tumors, and can regulate tumor cell growth, differentiation, invasion, and metastasis (Wong et al., 2009; Cui et al., 2014; Gao et al., 2014). Nevertheless, the role of miR-184 in osteosarcoma cells remains unclear. We evaluated the expression and function of miR-184 in osteosarcoma cells to identify new osteosarcoma markers and therapeutic targets.

\section{MATERIAL AND METHODS}

\section{Instruments and reagents}

The osteosarcoma cell line, SOSP-M, was bought from ATCC cell bank (Manassas, VA, 
USA). Transwell chambers, Dulbecco's modified Eagle medium (DMEM) and penicillin-streptomycin were purchased from Hyclone (Logan, UT, USA). Fetal bovine serum (FBS) was purchased from Hangzhou Sijiqing Co., Ltd. (Zhejiang, China). Trypsin-EDTA, 3-(4,5-Dimethylthaizol-2-yl)-2,5diphenyltetrazolium bromide (MTT), and dimethylsulfoxide (DMSO) were obtained from Sigma (St. Louis, MO, USA). The miR-184 mimetic and inhibitor were synthesized by Genepharma Co. Ltd., (Shanghai, China). The RNA extraction kit, reverse transcription kit, and Lipo2000 were from Invitrogen (Grand Island, NY, USA). DNA amplification was performed using the PE Gene Amp PCR System 2400 (Foster City, CA, USA).

\section{Methods}

\section{SOSP-M cell culture}

SOSP-M cells were revived in a $37^{\circ} \mathrm{C}$ water bath. After centrifugation at $1000 \mathrm{rpm}$ for 3 min, the cells were resuspended in $1 \mathrm{~mL}$ medium and maintained at $37^{\circ} \mathrm{C}$ in $5 \% \mathrm{CO}_{2}$. Cells $(1 \mathrm{x}$ $10^{7}$ ) were cultured in high glucose DMEM with $10 \%$ FBS and penicillin-streptomycin. Cells in the logarithmic growth phase were used for all experiments. The cells were divided into three groups: normal control, miR-184 mimic, and miR-184 inhibitor.

\section{Cell transfection}

MiR-184 mimic sequence: 5'-GGCAUUCUGUAUACAUCGGAG-3'; miR-184 inhibitor sequence: 5'-GAUCGGAGGUGCAUUCUA-3'. The miR-184 mimic and inhibitor were mixed with $200 \mu \mathrm{L}$ serum free medium. After incubation at room temperature for $15 \mathrm{~min}$, the medium was incorporated with Lipo2000 at room temperature for $30 \mathrm{~min}$. After washing with PBS, the cells were incubated with medium and maintained at $37^{\circ} \mathrm{C}$ and $5 \% \mathrm{CO}_{2}$ for $6 \mathrm{~h}$.

\section{Real-time PCR}

Total RNA was extracted from the cells using Trizol. The cDNA was synthesized using the reverse transcription kit. The primers used were designed by PrimerPrimier 6.0 and synthesized by Sangon (Shanghai, China). The primers were as follows: miR-184 sense: 5'-TACGACTATGTGACCTGCCTG-3'; anti-sense: 5'-TGGTTCAACTCTCCTTTCCA-3'; GAPDH sense: 5'-ATCTGGAGTTTACCGCTGG-3'; Anti-sense: 5'-TACCGATGTCTGGTAGACGAT-3'.

The cycling conditions were: 40 cycles of $60 \mathrm{~s}$ at $55^{\circ} \mathrm{C}, 30 \mathrm{~s}$ at $92^{\circ} \mathrm{C}, 45 \mathrm{~s}$ at $58^{\circ} \mathrm{C}$, and $35 \mathrm{~s}$ at $72^{\circ} \mathrm{C}$. Gene expression levels were quantified using an optimized comparative $\mathrm{Ct}(\Delta \Delta \mathrm{Ct})$ value method.

\section{MTT assay}

SOSP-M cells $\left(5 \times 10^{3}\right)$ were plated in 96 -well plates. After $24 \mathrm{~h}$ incubation, the cells were divided into three groups. MTT $(20 \mu \mathrm{l})$ was added at different time points. After $4 \mathrm{~h}$, MTT solution was removed and DMSO was added. The absorbance at $570 \mathrm{~nm}$ was read on a microplate reader (Synergy $^{\mathrm{TM}} 2$ Multi-Mode Microplate Reader, BioTek, Winooski, VT, USA). The experiments were performed in triplicate, and the cell proliferation rate was calculated. 


\section{Transwell assay}

After $48 \mathrm{~h}$ transfection, cells were seeded in Transwell chambers coated with Matrigel. After $48 \mathrm{~h}$ incubation, the chambers were fixed with ice-cold ethanol and stained with crystal violet for $30 \mathrm{~min}$. Cells that migrated to the lower side of the membrane were counted in 10 fields. The experiment repeated three times.

\section{Statistical analysis}

All statistical analyses were performed using SPSS13.0 software (Chicago, IL, USA). Numerical data were presented as the mean and standard deviation $( \pm S D)$. Differences between means were analyzed using one-way ANOVA or paired t-test. $\mathrm{P}$ values less than 0.05 were considered statistically significant.

\section{RESULTS}

\section{MiR-184 expression in SOSP-M cells}

The miR-184 mimic and inhibitor were transfected to SOSP-M cells using Lipo2000. Realtime PCR was used to detect miR-184. Compared to normal control cells, miR-184 expression was significantly increased in the miR-184 mimic group $(P<0.05)$. However, miR-184 inhibitor expression obviously reduced miR-184 expression $(P<0.05)$ (Figure 1$)$.

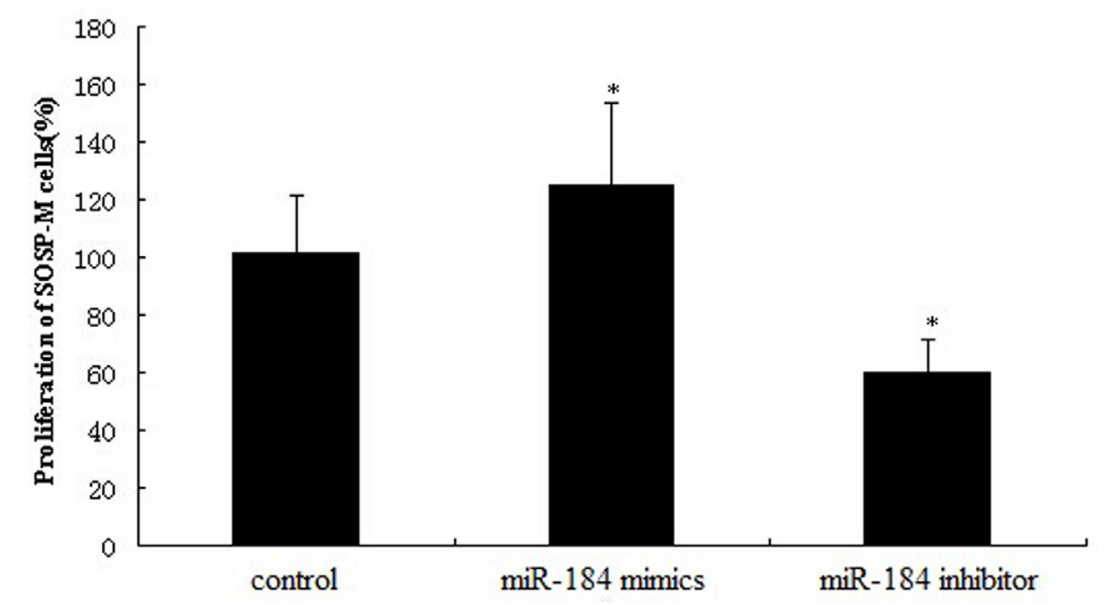

Figure 1. MiR-184 expression in SOSP-M cells. ${ }^{*} \mathrm{P}<0.05$ compared with control.

\section{Effect of miR-184 on SOSP-M cell proliferation}

The MTT assay was used to evaluate osteosarcoma cell proliferation. Tumor cell proliferation was enhanced in cells expressing the miR-184 mimic $(P<0.05)$, whereas miR-184 inhibition suppressed cell proliferation $(P<0.05)$ (Figure 2). 


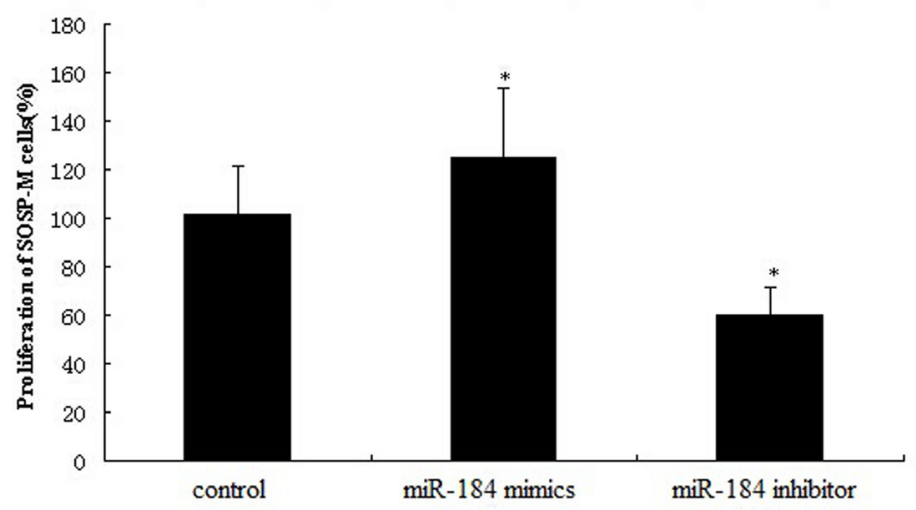

Figure 2. Effect of miR-184 on SOSP-M cell proliferation. ${ }^{*} \mathrm{P}<0.05$ compared with control.

\section{Effect of miR-184 on SOSP-M cell invasion}

A Transwell assay was performed to determine cell invasion. Tumor cell invasion increased after miR-184 mimic transfection $(P<0.05)$, and decreased after miR-184 was inhibited $(P<0.05)$ (Figures 3 and 4).
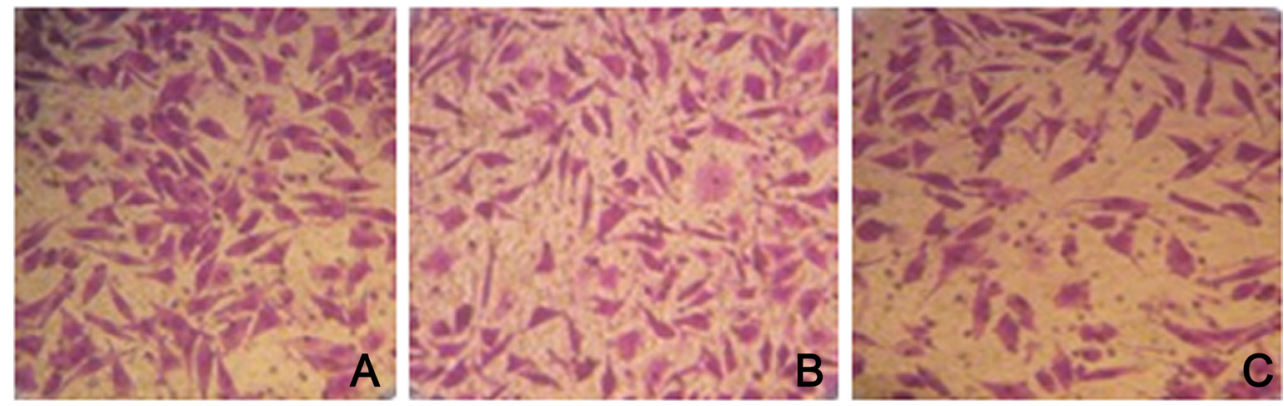

Figure 3. Effect of miR-184 on SOSP-M cell invasion.

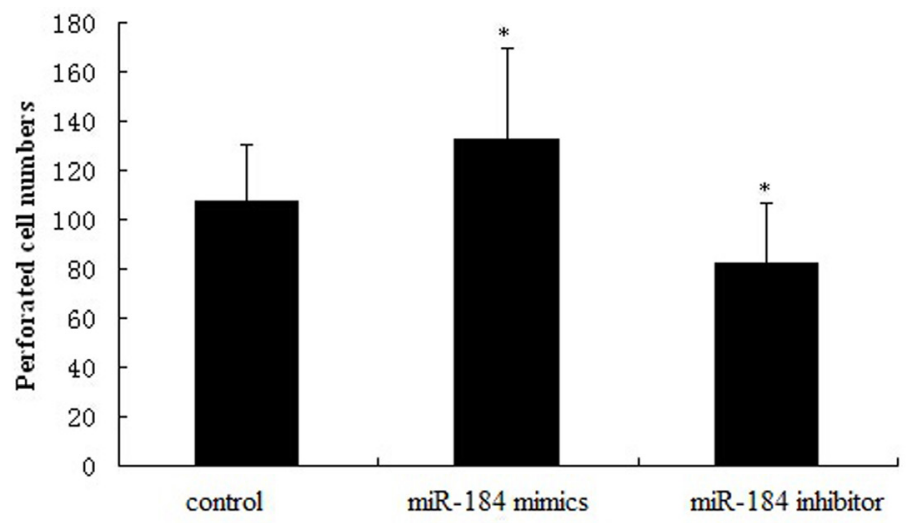

Figure 4. Effect of miR-184 on SOSP-M cell invasion. ${ }^{*} \mathrm{P}<0.05$ compared with control. 


\section{DISCUSSION}

Osteosarcoma is a highly aggressive primary bone tumor that poses numerous problems to orthopedic and oncology doctors, due to its quick progression, frequent metastasis, lack of treatment efficacy, and poor clinical prognosis. Genetic abnormalities contribute to tumor development, including normal gene overexpression, mutation, or deficiency, or oncogene expression. Interactions between related genes and the immune system regulate tumor protein expression (Huang and Ouyang, 2015). MiRNAs were first discovered in Caenorhabditis elegans, as Lin-4 can regulate nematode sequential development. Subsequent studies identified miRNAs in rice, fruit flies, viruses, nematodes, mice, and humans. Currently, over 4500 miRNAs have been identified. MiRNAs can post-transcriptionally inhibit gene expression, based on sequence specificity. They regulate cell growth, proliferation, differentiation, metabolism, and apoptosis. In general, miRNAs are composed of 19-25 nucleotides. Science magazine considered the discovery of miRNA to be one of the top ten scientific advancements in 2002 and 2003 (Garajova et al., 2014; Luo et al., 2014). The relationship between miRNAs and tumors was first identified in chronic lymphocytic leukemia (CLL). MiRNA microarray detection suggested that miR-15a and miR-16-1 were decreased in CLL patients, as compared to healthy controls. MiRNA microarrays revealed that changes in miRNA expression depended on tumor type. MiR-155 and miR-21 may negatively regulate cancer-suppressor genes and the cell cycle to promote cancer pathogenesis, whereas let-7, miR-15/16, and miR-34 negatively regulate oncogenes to inhibit tumor development. MiRNA expression and biological function have been studied in various tumors. MiRNA microarrays have clarified their relationship with tumor occurrence, development, and differentiation, and in favor of tumor diagnosis and classification (Li et al., 2015; Pei et al., 2015).

In view of their important roles in tumors, miRNAs are widely considered a biomarker of hematological and tumor tissues. MiRNAs may participate in bone formation through Twist, Hedgehog, fibroblast growth factors (FGF), and bone morphogenetic proteins (BMP) signaling. MiRNAs are abnormally expressed during osteosarcoma pathogenesis. Multiple genes were upregulated or downregulated, as determined by miRNA microarray. MiRNA expression changes before and after tumor treatment can be used to detect chemotherapy sensitivity (Zhao et al., 2014; Xiao et al., 2015). MiR-184 is a novel miRNA, and its role in tumors remains unclear. Studies show that it is abnormally expressed in diverse tumors and may regulate tumorigenesis. MiR-184 was overexpressed in glioma, suggesting that it promotes glioma pathogenesis. Furthermore, miR-184 upregulation significantly promotes glioma invasion (Wong et al., 2009; Cui et al., 2014; Gao et al., 2014). However, its role in osteosarcoma remains unknown. This study suggested that miR-184 overexpression promotes SOSP-M cell proliferation, whereas miR-184 inhibition obviously impedes SOSP-M cell proliferation. Tumor cells invasiveness increased after microRNA184 mimic transfection and decreased after miR184 inhibition. These data indicate that miR-184 is directly associated with osteosarcoma occurrence and development, and participates in osteosarcoma metastasis.

In conclusion, miR-184 participates in the regulation of osteosarcoma development and metastasis, and may represent a novel clinical marker for osteosarcoma diagnosis and therapeutic target for metastatic osteosarcoma. Further evaluation of its mechanism in osteosarcoma is required.

\section{Conflicts of interest}

The authors declare no conflict of interest. 


\section{REFERENCES}

Cui QK, Liu WD, Zhu JX, Wang YH, et al. (2014). MicroRNA-184 promotes proliferation ability of glioma cells by regulating FOXO3. Asian Pac. J. Trop. Med. 7: 776-779.

Ebrahimzadeh MH, Vahedi E, Ganji R and Bozorgnia S (2013). Skeletal sarcoma on the site of retained war bullet fragments and a literature review on long-term complications of retained war shells. Arch. Bone Jt Surg. 1: 107-111.

Gallach S, Calabuig-Fariñas S, Jantus-Lewintre E and Camps C (2014). MicroRNAs: promising new antiangiogenic targets in cancer. Biomed. Res. Int. 2014: 878450.

Gandhi NS, Tekade RK and Chougule MB (2014). Nanocarrier mediated delivery of siRNA/miRNA in combination with chemotherapeutic agents for cancer therapy: current progress and advances. J. Control Release 194: 238-256.

Gao B, Gao K, Li L, Huang Z, et al. (2014). miR-184 functions as an oncogenic regulator in hepatocellular carcinoma (HCC). Biomed. Pharmacother. 68: 143-148.

Garajova I, Le Large TY, Frampton AE, Rolfo C, et al. (2014). Molecular mechanisms underlying the role of microRNAs in the chemoresistance of pancreatic cancer. Biomed. Res. Int. 2014: 678401.

Huang $Q$ and Ouyang X (2015). The role of miRNAs in bone metastasis and their significance in the detection of bone metastasis: a review of the published data. Future Oncol. 11: 141-151.

Li H, Liu H, Pei J, Wang H, et al. (2015). miR5423p overexpression is associated with enhanced osteosarcoma cell proliferation and migration ability by targeting Van Goghlike 2. Mol. Med. Rep. 11: 851-856.

Lindner K, Haier J, Wang Z, Watson DI, et al. (2015). Circulating microRNAs: emerging biomarkers for diagnosis and prognosis in patients with gastrointestinal cancers. Clin. Sci. 128: 1-15.

Luo XJ, Tang DG, Gao TL, Zhang YL, et al. (2014). MicroRNA-212 inhibits osteosarcoma cells proliferation and invasion by down-regulation of Sox4. Cell Physiol. Biochem. 34: 2180-2188.

Orang AV and Barzegari A (2014). MicroRNAs in colorectal cancer: from diagnosis to targeted therapy. Asian Pac. J. Cancer Prev. 15: 6989-6999.

Pei H, Jin Z, Chen S, Sun X, et al. (2015). MiR-135b promotes proliferation and invasion of osteosarcoma cells via targeting FOXO1. Mol. Cell Biochem. 400: 245-252.

Phuah NH and Nagoor NH (2014). Regulation of microRNAs by natural agents: new strategies in cancer therapies. Biomed. Res. Int. 2014: 804510.

Saadatian Z, Masotti A, Nariman Saleh Fam Z, Alipoor B, et al. (2014). Single-nucleotide polymorphisms within microRNAs sequences and their $3^{\prime}$ UTR target sites may regulate gene expression in gastrointestinal tract cancers. Iran Red. Crescent Med. J. 16: e16659.

Sun L, Li Y, Zhang J, Li H, et al. (2015). Prognostic value of pathologic fracture in patients with high grade localized osteosarcoma: a systemic review and meta-analysis of cohort studies. J. Orthop. Res. 33: 131-139.

Trosman SJ and Krakovitz PR (2015). Pediatric maxillary and mandibular tumors. Otolaryngol. Clin. North Am. 48: 101-119.

Vijayakumar V, Lowery R, Zhang X, Hicks C, et al. (2014). Pediatric osteosarcoma: a single institution's experience. South Med. J. 107: 671-675.

Wong TS, Ho WK, Chan JY, Ng RW, et al. (2009). Mature miR-184 and squamous cell carcinoma of the tongue. ScientificWorldJournal 9: 130-132.

Xiao F, Chen J, Lian C, Han P, et al. (2015). Tumor necrosis factor-related apoptosis-inducing ligand induces cytotoxicity specific to osteosarcoma by microRNA response elements. Mol. Med. Rep. 11: 739-745.

Zhao M, Huang J, Gui K, Xiong M, et al. (2014). The downregulation of miR-144 is associated with the growth and invasion of osteosarcoma cells through the regulation of TAGLN expression. Int. J. Mol. Med. 34: 1565-1572. 